\title{
ARTICLE OPEN Chronic social defeat stress impairs goal-directed behavior through dysregulation of ventral hippocampal activity in male
} mice

\author{
Keitaro Yoshida ${ }^{1}$, Michael R. Drew (D) ${ }^{2}$, Anna Kono ${ }^{1}$, Masaru Mimura ${ }^{1}$, Norio Takata ${ }^{1}{ }^{1}$ and Kenji F. Tanaka (D)
}

\begin{abstract}
Chronic stress is a risk factor for a variety of psychiatric disorders, including depression. Although impairments to motivated behavior are a major symptom of clinical depression, little is known about the circuit mechanisms through which stress impairs motivation. Furthermore, research in animal models for depression has focused on impairments to hedonic aspects of motivation, whereas patient studies suggest that impairments to appetitive, goal-directed motivation contribute significantly to motivational impairments in depression. Here, we characterized goal-directed motivation in repeated social defeat stress (R-SDS), a wellestablished mouse model for depression in male mice. R-SDS impaired the ability to sustain and complete goal-directed behavior in a food-seeking operant lever-press task. Furthermore, stress-exposed mice segregated into susceptible and resilient subpopulations. Interestingly, susceptibility to stress-induced motivational impairments was unrelated to stress-induced social withdrawal, another prominent effect of R-SDS in mouse models. Based on evidence that ventral hippocampus (vHP) modulates sustainment of goal-directed behavior, we monitored vHP activity during the task using fiber photometry. Successful task completion was associated with suppression of ventral hippocampal neural activity. This suppression was diminished after R-SDS in stress-susceptible but not stress-resilient mice. The serotonin selective reuptake inhibitor (SSRI) escitalopram and ketamine both normalized vHP activity during the task and restored motivated behavior. Furthermore, optogenetic vHP inhibition was sufficient to restore motivated behavior after stress. These results identify vHP hyperactivity as a circuit mechanism of stress-induced impairments to goal-directed behavior and a putative biomarker that is sensitive to antidepressant treatments and that differentiates susceptible and resilient individuals.
\end{abstract}

Neuropsychopharmacology (2021) 46:1606-1616; https://doi.org/10.1038/s41386-021-00990-y

\section{INTRODUCTION}

Impaired motivation is a cardinal symptom of depression [1, 2]. Among depression symptoms, diminished motivational drive is one of the strongest predictors of a depression diagnosis [3]. Motivational impairments potentially involve perturbations to at least two distinct motivational processes: changes in the hedonic impact (liking or pleasure) derived from rewards (RWs), and changes in cost-benefit calculations that control the willingness to engage in and sustain effortful behaviors required to earn a RW $[4,5]$. It can be difficult to disentangle these processes experimentally [6]. Although there is indirect evidence suggesting that hedonic responses to RW are blunted in depressed patients, studies directly assessing pleasure responses have not yielded evidence of impaired hedonic impact in depressed patients [7]. In contrast, depressed patients do exhibit deficits in effortful RWbased tasks [8], leading some researchers to conclude that motivational deficits in depressed patients reflect a decreased willingness to expend effort to obtain RWs [9].

Despite this evidence from patient studies, research in animal models for depression has tended to focus on hedonic RW. In two well-validated rodent models for depression, chronic social defeat stress (SDS) [10] and chronic unpredictable stress [11], stress- exposed animals display a reduced preference for sweet RWs, which has been interpreted as reflecting blunted hedonic impact. Studies linking stress and motivation have typically focused on striatal regions implicated in hedonic RW processing [12]. There is also evidence that chronic stress can impair goal-directed motivation in operant tasks that require sustained responding to earn RW $[13,14]$. However, the mechanisms of stress effects on goal-directed motivation are unknown.

Recent evidence, however, identifies vHP, a region believed to be highly sensitive to both stress and antidepressant medications [15-19], as a critical regulator of goal-directed motivation. In tasks that require sustained effort to earn a RW, ventral CA1 (vCA1) population activity is suppressed during periods of sustained effort, and optogenetic manipulations demonstrate that this suppression is required for successful completion of the task [20]. Based on prior evidence linking chronic stress to hippocampal hyperactivity $[21,22]$, we hypothesized that chronic stress exposure might impair goal-directed motivation by interfering with the suppression of vCA 1 activity.

Although stress is a major risk factor for mood disorders [23], there is considerable inter-individual variation in the susceptibility versus resilience to stress [24]. This heterogeneity has been

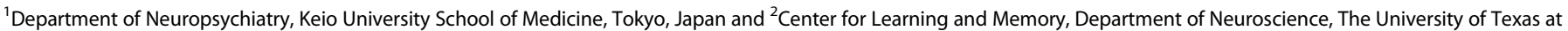
Austin, Austin, TX, USA

Correspondence: Kenji F. Tanaka (kftanaka@keio.jp)
}

Received: 17 November 2020 Revised: 30 January 2021 Accepted: 20 February 2021

Published online: 10 March 2021 
modeled in animals using the chronic SDS paradigm [10]. In mice, exposure to chronic SDS induces social withdrawal, but among stress-exposed animals there are susceptible and resilient populations. In recent years considerable effort has been directed at identifying neural and molecular mechanisms of stress susceptibility and resilience $[25,26]$. It remains unclear, however, whether stress effects on motivation are equally heterogeneous and whether the mechanisms controlling motivational resilience and susceptibility are the same as those mediating stress effects on social behavior.

Here, we demonstrate that social defeat stress (R-SDS) impairs goal-directed motivation in male mice. However, the population of stress-exposed animals can be stratified into susceptible and resilient populations. Using fiber photometric recordings of bulk neural activity, we show that stress effects on goal-directed motivation are associated with perturbations in ventral hippocampal activity, and stress-induced motivational impairments can be alleviated with appropriately timed manipulation of vHP activity. Finally, we demonstrate that two distinct classes of antidepressants, a slow-acting serotonin selective reuptake inhibitor (SSRI) and rapid/prolonged-acting ketamine, rescue stress-induced impairments in motivated behavior and hippocampal function.

\section{METHODS AND MATERIALS}

Animals

All animal procedures were conducted in accordance with the National Institutes of Health Guide for the care and use of laboratory animals and approved by the Animal Research Committee of Keio University. Experiments were carried out using 8- to 14-week-old male mice. All mice were maintained with 12:12$\mathrm{h}$ light/dark cycle (lights on at $8 \mathrm{am}$ ) and the behavioral experiments were conducted during the light phase. $\mathrm{Htr} 5 \mathrm{~B}-\mathrm{YC}$ mice (Htr5B-tTA::tetO-YCnano50 double transgenic mice) were obtained by crossing Htr5B-tTA mice [27] and tetO-YCnano50 mice [28]. Htr5B-ArchT mice (Htr5B-tTA::tetO-ArchT-EGFP double transgenic mice) were obtained by crossing Htr5B-tTA mice and tetOArchT-EGFP mice [29]. The genetic background of all transgenic mice was mixed C57BL6 and 129SvEvTac. Genotyping for Htr5BtTA, tetO-YCnano50, and tetO-ArchT was previously described [27-29]. Male ICR mice over 13 weeks of age were purchased from Oriental Yeast Co, Ltd, Japan.

\section{Surgical procedure}

Surgeries were performed using a stereotaxic system (SM-6M-HT, Narishige). Mice were anesthetized with a mixture of ketamine and xylazine $(100 \mathrm{mg} / \mathrm{kg}$ and $10 \mathrm{mg} / \mathrm{kg}$, respectively, intraperitoneal [i.p.]). Their body temperature was maintained at $37 \pm 0.5^{\circ} \mathrm{C}$ using a heating pad (FHC-MO, Muromachi Kikai) during surgery. For optical recordings at the vCA1, Htr5B-YC mice were unilaterally (right side or left side) implanted with an optical fiber cannula (CFMC14L05, $400 \mu \mathrm{m}, 0.39 \mathrm{NA}$; Thorlabs) into the vCA1 (-3.08 mm anteroposterior (AP), $3.5 \mathrm{~mm}$ mediolateral (ML) from bregma, 3.7 $\mathrm{mm}$ dorsoventral (DV) from the brain surface) according to the atlas of Paxinos and Franklin [30]. For optogenetic manipulations, Htr5B-ArchT mice were bilaterally implanted with $200 \mu \mathrm{m}$ core diameter optical fiber (0.39 NA, Thorlabs) into the vCA1 $(-3.08$ $\mathrm{mm} \mathrm{AP}, \pm 3.5 \mathrm{~mm}(\mathrm{ML}), 3.7 \mathrm{~mm} \mathrm{DV})$.

\section{Fixed ratio (FR) operant task}

The method for the food-seeking lever-press task has been described previously [20]. Mice were housed individually under conditions of food restriction. Their body weights were maintained at $85 \%$ of initial body weight. Behavioral trainings and tests were performed in an aluminum operant chamber measuring W22 $\times$ D $26 \times \mathrm{H} 18 \mathrm{~cm}$ (Med Associates) under constant darkness. The apparatus was controlled by a computer program written in the MED-PC language (Med Associates). A food dispenser flanked by two retractable levers was located on the floor. The lever on the left side is designated as "active" (triggering delivery of a food RW), and the one on the right is "inactive" (no relation to food RW). Each trial began with the presentation of two levers (trial start [TS]). After mice pressed the active lever (lever press [LP]), the levers were retracted and one food pellet $(20 \mathrm{mg}$ each, dustless precision pellets, Bio-serv) was delivered as a RW. After the food delivery, $30 \mathrm{~s}$ of intertrial interval (ITI) was added, during which levers were retracted, followed by the automatic starting of the next trial. The ITI allows time for mice to consume the food pellet. The training started with a FR-1 schedule, in which the mice obtained one food RW after each active LP. Once the animals were able to obtain $50 \mathrm{RWs}$ within $60 \mathrm{~min}$, the training progressed to an FR-2 schedule, in which two active LPs were needed for a couple of sessions. The training moved on to an FR-3 schedule when the animals could obtain 50 trial RWs in 60 min. Each FR training session lasted $1 \mathrm{~h}$ or when 100 pellets had been delivered. In an FR-5 or FR-10, the lever presentation was lasted for $30 \mathrm{~s}$. If the mice pressed performed the required number of active LPs during the lever presentation, the levers were retracted and one food pellet was delivered ("successful trial"). After the food delivery, there was a 30-s ITI, during which levers were retracted, followed by the automatic starting of the next trial. If the mouse pressed the active lever at least once, but did not make the required number of presses during lever presentation, the levers were retracted and trial ended ("incomplete trial"). Incomplete trials were not included in the analysis of completion latency. If the mouse never pressed the active lever during lever presentation, the levers were retracted and the trial ended ("omission trial"). On average, it took 20 days for surgery, recovery, and the entire behavioral procedure including training.

\section{Fiber photometry}

The method for fiber photometry has been described previously [31]. An exciting light ( $435 \mathrm{~nm}$; silver-LED, Prizmatix) was reflected off a dichroic mirror (DM455CFP; Olympus), focused with a $20 \times$ objective lens (NA 0.39, Olympus), and coupled into an optical fiber (M79L01, $\Phi 400 \mu \mathrm{m}, 0.39 \mathrm{NA}$; Thorlabs) through a pinhole $(\Phi$ $400 \mu \mathrm{m})$. LED power was $<100 \mu \mathrm{W}$ at the fiber tip. Emitted cyan and yellow fluorescence from YCnano 50 was collected via an optical fiber cannula, divided by a dichroic mirror (DM515YFP; Olympus) into cyan (483/32 nm band-path filters, Semrock) and yellow $(542 / 27 \mathrm{~nm})$, and detected by each photomultiplier tube (H10722-210, Hamamatsu Photonics). The fluorescence signals in addition to $T L$ signals from behavioral settings were digitized by a data acquisition module (cDAQ-9178, National Instruments), and simultaneously recorded using a custom-made LabVIEW program (National Instruments). Signals were collected at a sampling frequency of $1000 \mathrm{~Hz}$.

\section{Optogenetic manipulation during operant task}

The optogenetic methods have been described previously [20]. Two sessions were conducted with stimulation (yellow) or control (blue), counterbalanced. Optical fibers (NA 0.39, Thorlabs) were inserted bilaterally through the guide cannulae. Yellow $(575 \mathrm{~nm})$ and blue $(475 \mathrm{~nm})$ light were generated by a SPECTRA 2-LCR-XA light engine (Lumencor). The yellow and blue light power intensities at the tip of the optical fiber were 3-4 $\mathrm{mW}$ and 2-3 $\mathrm{mW}$, respectively. The light was controlled by the $T \mathrm{~L}$ pulses generated by MED-PC (Med Associates). In FR task, one session, consisted of 50 trials (stimulation; 25 trials, control; 25 trials, counterbalanced), was conducted.

Repeated social defeat stress (R-SDS)

Social defeat (SD) mice were exposed to chronic SDS as previously described [32]. We used only male mice because aggressor mice do not attack female mice if there is no genetic manipulation 
introduced in the aggressor mice. At least 1 week before beginning the social defeat experiment, all resident ICR mice more than 13 weeks of age were singly housed on home cage $(18.2 \mathrm{~cm} \times 26.0 \mathrm{~cm} \times 12.8 \mathrm{~cm})$. Prior to R-SDS, male ICR mice were screened for their aggressiveness to a novel C57BL/6J mouse for 3 min daily for 3 days. We evaluated the aggression of the ICR mice by the latency and the number of attacks during the observation period, and only used those showing stable aggression for further experiments. After evaluating behavioral parameters and vCA1 activity of test mice, we conducted R-SDS. These mice were then transferred to the home cage of a male ICR mouse for 20 min daily for 10 days. The pairs of defeated and aggressor mice were randomized daily to minimize the variability in the aggressiveness of aggressor mice. After the 20 min defeat episode, the mice were returned to their home cages and kept isolated until SDS on the next day. During R-SDS phase, we conducted the FR task daily.

Escitalopram administration

Escitalopram (15 mg/kg per day in deionized water) or vehicle was delivered by oral gavage of for 3 weeks after R-SDS procedures [33]. On the days when mice were subjected to the FR task, escitalopram or vehicle administrations were conducted after the mice completed the testing. During the escitalopram treatment phase, these mice were tested on the FR task daily.

\section{(R,S)-Ketamine administration}

Mice received a single acute intraperitoneal injection of vehicle or $(\mathrm{R}, \mathrm{S})$-ketamine. Based on previous studies [34], the dose was 10 $\mathrm{mg} / \mathrm{kg}$. Followed by $24 \mathrm{~h}$, we conducted the FR task to evaluate the effect of $(R, S)$-ketamine. We used only $(R, S)$-ketamine.

\section{Social interaction (SI) test}

SI tests were performed inside a dark room with a light intensity of 10 lux in a square-shaped box $(40 \mathrm{~cm} \times 40 \mathrm{~cm})$ enclosed by walls $27 \mathrm{~cm}$ in height. A wire mesh cylindrical cage $(8.0 \mathrm{~cm}$ diameter $\times$ $24 \mathrm{~cm}$ high) was centered against one wall of the arena during all $\mathrm{SI}$ sessions. Each SI test included two $150 \mathrm{~s}$ sessions (separated by an intersession interval of $30 \mathrm{~s}$ ) without and with the target ICR mouse present in the mesh cage; these sessions were termed "notarget" and "target" sessions, respectively. In the no-target session, a test mouse was placed in the box and allowed to freely explore the environment. The mouse was then removed from the box. In the $30 \mathrm{~s}$ break between sessions, the target ICR mouse was introduced into the mesh cage. The design of the cage allowed the animal to fit its snout and paws through the mesh cage but not to escape from the cage. In the target session, the same test mouse was placed beside the wall opposite to the mesh cage. In each session, the time spent in the interaction zone, extending $8 \mathrm{~cm}$ around the mesh cage was analyzed. The SI ratio was computed as the ratio of time spent in the interaction zone in the presence of the target to the time spent there in the absence of the target.

\section{Immunohistochemistry}

Following completion of each experiment, mice were deeply anesthetized with ketamine $(100 \mathrm{mg} / \mathrm{kg})$ and xylazine $(10 \mathrm{mg} / \mathrm{kg})$ and perfused with $4 \%$ paraformaldehyde phosphate-buffer solution. Brains were removed from the skull and postfixed in the same fixative overnight. Subsequently, brains were cryoprotected in $20 \%$ sucrose overnight, frozen, and cut at $25 \mu \mathrm{m}$ thickness on a cryostat. Sections were mounted on silane-coated glass slides (Matsunami Glass). Sections were incubated with the primary antibodies overnight at room temperature. The following antibodies were used: anti-green fluorescent protein (GFP) (1:200, goat polyclonal, Rockland Immunochemicals Inc.). For fluorescence microscopy, sections were treated with species-specific secondary antibodies conjugated to Alexa Fluor 488 (1:1,000, Invitrogen) for $2 \mathrm{~h}$ at room temperature. Fluorescent images were obtained with an all-in-one microscope (BZ-X710, Keyence).

\section{Data analysis}

All animals and samples were randomly assigned to the experimental groups. Data collection and analysis were not performed blind to the conditions of the experiments. When optic fiber position, electrode position, or microinjection cannula position was not targeted correctly, we excluded those mice. Fiber photometry data were analyzed using custom-written programs in MATLAB. YC ratio (a ratio of yellow to cyan fluorescence intensity; $R$ ) in one session was detrended using cubic spline, and normalized within each trial by calculating the $Z$-score as $(R-$ $\left.R_{\text {mean }}\right) / R_{\mathrm{SD}}$, where $R_{\text {mean }}$ and $R_{\mathrm{SD}}$ were the mean and standard deviation of the YC ratio for $5 \mathrm{~s}$ just prior to each TS.

\section{Statistics}

Photometry recordings and behavioral experiments were statistically analyzed using MATLAB and SPSS version 24 (IBM, USA). Normality and equal variances were formally tested. Two-sample comparisons were performed by two-sided unpaired $t$ test or twoway repeated-measures ANOVA followed by post hoc Bonferroni correction. If the data were not normally distributed, we performed with the nonparametric two-sided Wilcoxon signedrank test. Multiple group comparisons were performed by Mann-Whitney $U$-test and the significance was set at a familywise false discovery rate (FDR) adjusted $p$ value of 0.05 . k-means clustering was done using the MATLAB function kmeans. Data distribution was analyzed by Spearman's rank correlation test. Sample sizes were not predetermined, but our sample sizes are similar to ( $n$ animals) or higher than ( $n$ traces) those generally employed in the field $[20,35]$. All tests used are specified in the supplementary table.

\section{RESULTS}

Chronic social defeat stress impairs goal-directed behavior To monitor goal-directed motivated behavior, we used a foodseeking lever-press operant task. In this task, male mice were trained to press a lever five times to obtain one palatable pellet within $30 \mathrm{~s}$ (FR-5; Fig. 1a). We previously demonstrated that performance is sensitive to outcome devaluation [20], indicating that behavior is goal-directed in this task. Furthermore, behavioral performance (i.e., success rate) tracked the motivational state and was stable across time in individuals (Fig. 1c, d) [20]. To investigate the effects of stress in this task, we employed a R-SDS model, which is a validated pre-clinical model for major depressive disorder $[10,36]$. Each day for 10 consecutive days, we evaluated performance on the FR-5 task and then, immediately after the task, exposed each mouse to a larger aggressive mouse for $20 \mathrm{~min}$ (Fig. 1b). Latency to the first LP on each trial (the period from TS to 1 st LP) was used as a measure of action initiation. Latency was prolonged beginning 1 day after the start of SDS and remained so for all 10 days of R-SDS (Fig. 1c, top). The number of incomplete trials in which mice did not complete 5 LPs within $30 \mathrm{~s}$ gradually increased during R-SDS (Fig. 1d). Completion latency (LP to RW delivery [RD]), a measure of action sustainment, was also increased during R-SDS (Fig. 1c, middle). The total trial number and the success trial ratio were decreased, and the omission trial ratio was increased during R-SDS (supplementary Fig. S1). These data demonstrated that chronic stress exposure reduced motivated behavior. Food collection latency was not altered during R-SDS (Fig. 1c, bottom), suggesting that the preference for palatable pellets after stress exposure was comparable to that before stress exposure. The percentage of incomplete trials in the FR-5 task did not correlate with locomotor activity after R-SDS exposure (supplementary Fig. S2), suggesting that the reduction of FR-5 task performance was not attributable to general changes in activity.

Previous reports demonstrate that mice exposed to R-SDS can be divided into susceptible and resilient populations [10]. Indeed, 
Lever presentation period (30 s)

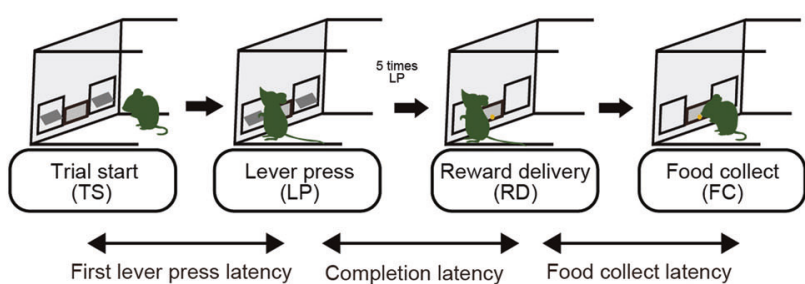

c
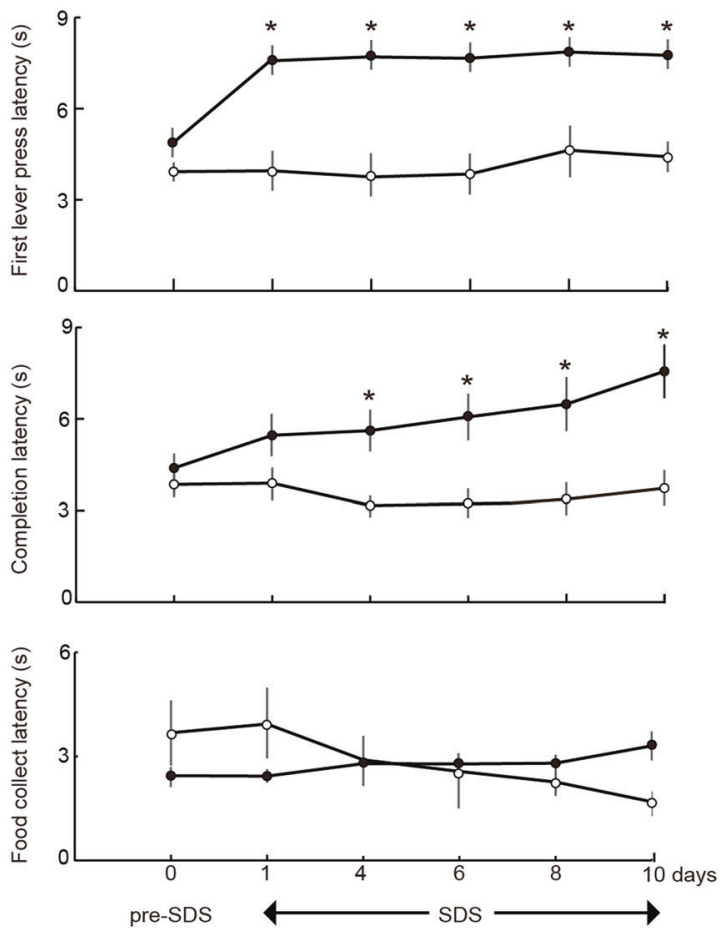

f
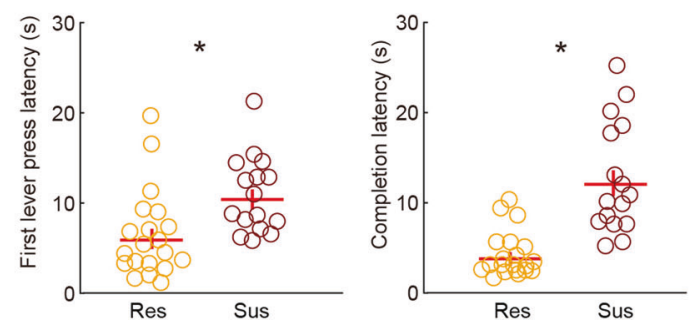

b Daily FR5 task with repeated social defeat stress (R-SDS)

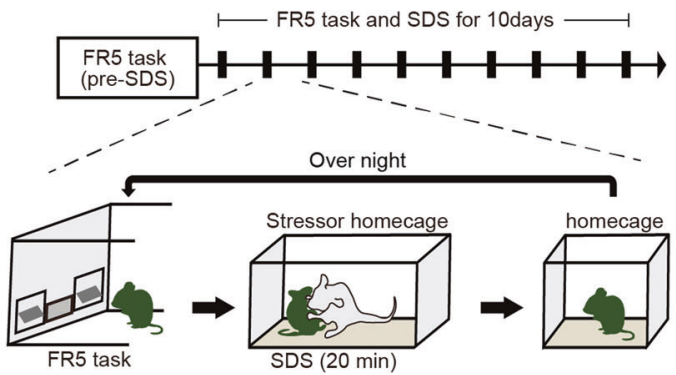

d
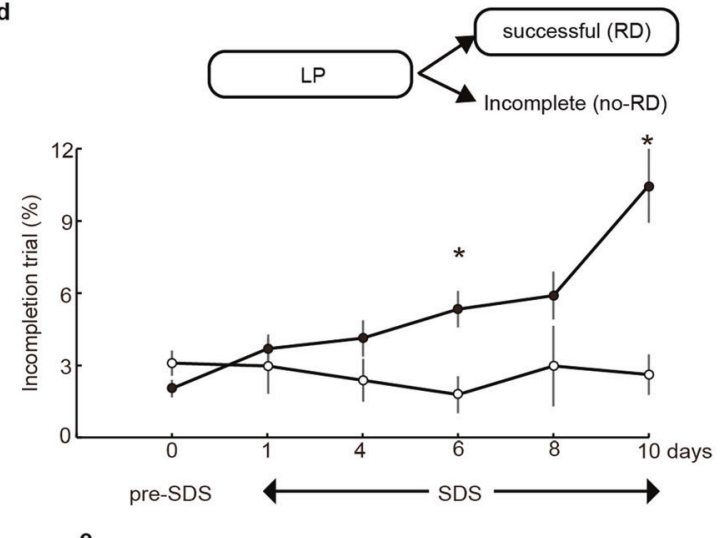

e

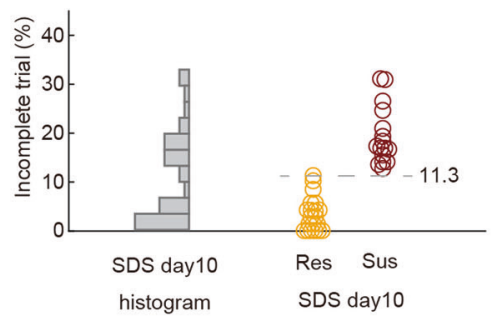

g

Fig. 1 Repeated social defeat stress impairs goal-directed behavior only in stress-susceptible mice. a Schematic illustration of the FR- 5 schedule. b Experimental schedule (upper) and schematic illustration (lower) of operant training during repeated social defeat stress (R-SDS). c The effects of repeated social defeat stress ( $n=36$, black circles) on first lever-press latency (top), completion latency of FR-5 (middle), and food collection latency (bottom) in the FR-5 task. Controls (without R-SDS, $n=12$, white circles) showed stable task performance. Two-way repeated-measures ANOVA confirmed significant group $\times$ session interactions on first lever-press latency $(p=0.004)$ and completion latency $(p=0.041)$ but not food collection latency $(p=0.737)$. Bars represents s.e.m. Asterisks $\left({ }^{*} p<0.01\right)$ represent significant $t$-test with Bonferroni correction (compared with the same time point). $\mathbf{d}$ Mice receiving R-SDS exhibited a gradual increase in incomplete trials in the FR-5 task $(n=$ 36 mice) compared with controls. Two-way repeated-measures ANOVA confirmed a significant group $\times$ session interactions on incomplete trials $(p=0.031) .{ }^{*} p<0.01, t$-test with Bonferroni correction. e Histogram of incomplete trial\% at SDS day 10 indicates a bimodal distribution. Defeated mice were categorized as susceptible $(n=16)$ or resilient $(n=20)$ based on incomplete trials $(\mathrm{k}$-means clustering, $k=2)$. Threshold value dividing susceptible and resilient groups was $11.3 \%$. f First lever-press latency and completion latency in susceptible mice were increased (resilient mice, $n=20$; susceptible mice, $n=16$ mice), but the food collection latency in FR-5 task was not changed. ${ }^{*} p<0.05$ ( $t$-test). g The percentage of incomplete trials did not correlate with SI ratio ( $n=36$ mice).

approximately half of R-SDS-exposed mice exhibited a decreased SI ratio as compared to mice that were not stressed (supplementary Fig. S3). We examined whether resilient and susceptible populations were also identifiable based on goal-directed motivated behavior. We classified defeated mice into two categories, susceptible and resilient, based on their percentage of incomplete trials using a k-means clustering algorithm with $k=$ 2 (Fig. 1e). The susceptible population exhibited increased 
latencies to the first LP and to task completion, whereas stress did not affect these measures in the resilient population (Fig. 1f). We next examined whether stress effects on social behavior predicted the effects of stress on motivated behavior. We found no significant correlations between $\mathrm{SI}$ ratio and behavioral parameters of the FR-5 task (Fig. 1g, Supplementary Fig. S4). In summary, results indicate that R-SDS decreases motivation on average, but the population includes susceptible and resilient subgroups. Furthermore, susceptibility to the stress-induced motivational impairments was unrelated to susceptibility to stress-induced social avoidance, suggesting that these two consequences of stress have distinct mechanisms.

Stress-susceptible mice exhibit increased ventral CA1 activity during goal-directed behavior

We recently demonstrated that the ventral hippocampal CA1 (vCA1) controls action sustainment during goal-directed behavior [20]. Accordingly, we examined the correspondence between vCA1 activity and behavioral change during R-SDS. To monitor
vCA1 activity in the task, we used transgenic mice harboring a $\mathrm{Ca}^{2+}$ indicator, Yellow Cameleon nano50 (YC), in hippocampal CA1 pyramidal neurons (Htr5B-YC mice, Fig. 2a, Supplementary Fig. S5) and applied fiber photometry $[20,31]$ to record compound $\mathrm{Ca}^{2+}$ signals from vCA1 in freely moving mice (Fig. 2b). Before stress exposure (no-SDS), the temporal pattern of vCA1 activity was as follows: vCA1 activity gradually decreased during the preparatory period (TS-LP), was sustained at the lowered level during lever pressing (LP-RD), rapidly increased after RD, and then overshot and returned to the baseline (Fig. 2c, e, black trace).

All Htr5B-YC mice were exposed R-SDS. We monitored vCA1 activity during the FR-5 task following the first SDS exposure (1 day after) (Fig. 2c, d, green trace) and following the 10th SDS exposure (10 day after) (Fig. 2e, f). Although the latency to the first LP was delayed following a single stress exposure (Fig. 1c), we did not find any effect of stress on VCA1 activity (Fig. 2c, d) at this time point, consistent with prior evidence that vCA1 activity does not modulate action initiation [20,35]. On the other hand, after 10 days of repeated stress exposure, we found significant changes
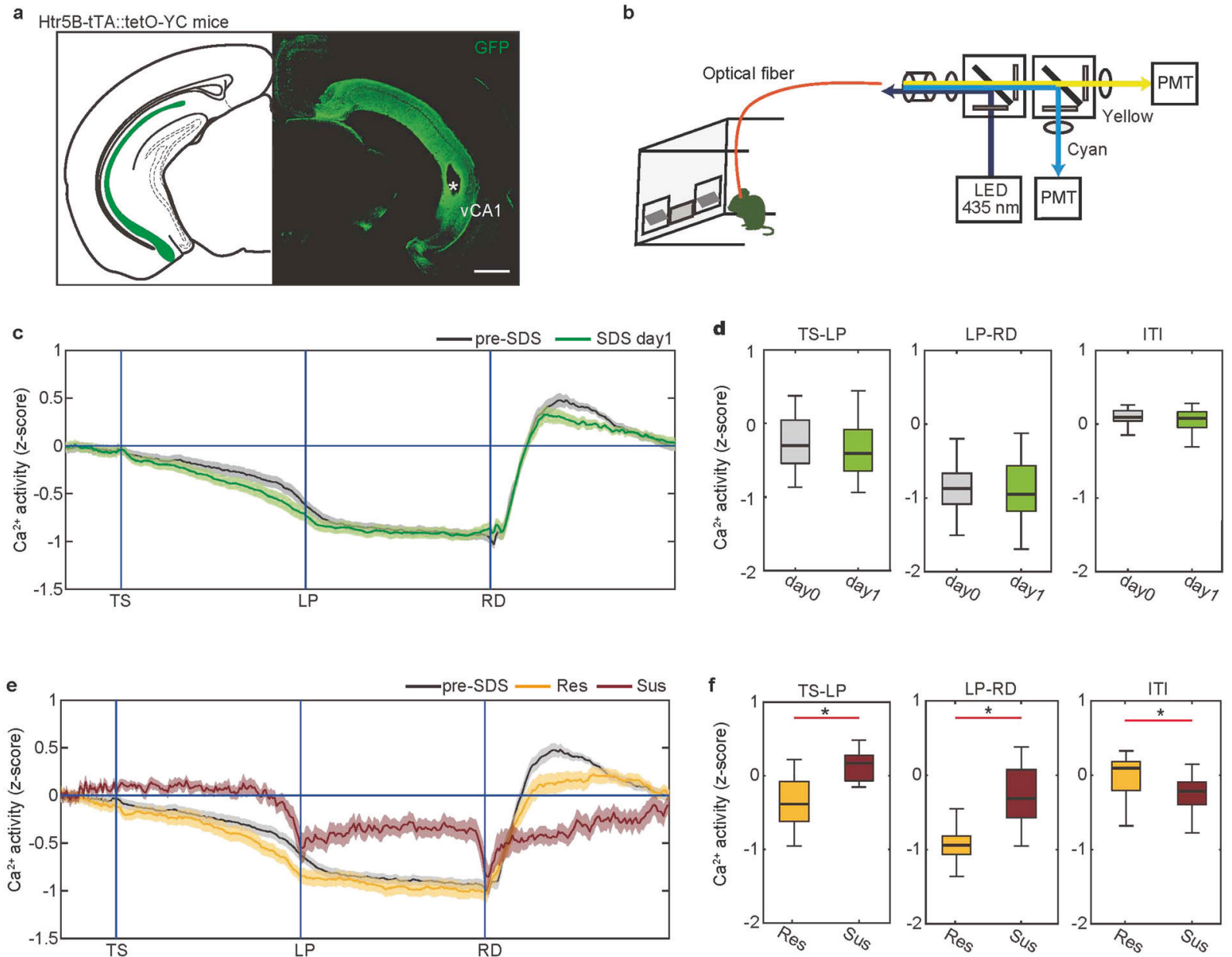

Fig. 2 Insufficient vCA1 suppression in mice susceptible to stress-induced motivational impairments. a YCnano50 expression (green) in the vCA1 of Htr5B-YC mice. The white asterisk denotes optical fiber placement. Scale bar, $1 \mathrm{~mm}$. b Ratiometric fiberphotometry system. YCnano50 was excited with $435 \mathrm{~nm}$. Cyan and yellow fluorescent signals were detected by respective PMTs. c Trace of averaged Ca ${ }^{2+}$ signals in the vCA 1 of Htr5B-YC mice ( $n=24$ mice) in which the duration between trigger points was normalized. vCA 1 activity during the FR-5 task 1 day after the first SDS exposure (day 1, green) did not differ from activity before stress exposure (day 0 , black). The shaded areas represent $\mathrm{S}$. e.m. d Boxplot representing the averaged $\mathrm{Ca}^{2+}$ signal during the TS-LP, LP-RD, and ITI periods of (c), respectively $(n=24$ mice). vCA1 activity in SDS day 1 was comparable to that in pre-SDS. In box plots, the central mark indicates the median and the bottom and top edges of the box indicate the 25 th and 75 th percentiles, respectively. Whiskers denote the range. e Trace of averaged $\mathrm{Ca}^{2+}$ signals in vCA1 of resilient (yellow, $n$ $=11$ mice) or susceptible mice in SDS day 10 (brown, $n=9$ mice) in which the duration between trigger points was normalized. The shaded areas represent s.e.m. The black trace shows pre-SDS in (c). f Boxplot representing the averaged $\mathrm{Ca}^{2+}$ signal during the TS-LP, LP-RD, and ITI period of (e), respectively (resilient, $n=11$ mice; susceptible, $n=9$ mice). vCA1 activity in susceptible mice was significantly elevated compared with resilient mice. ${ }^{*} p<0.05$ (t-test). 
a

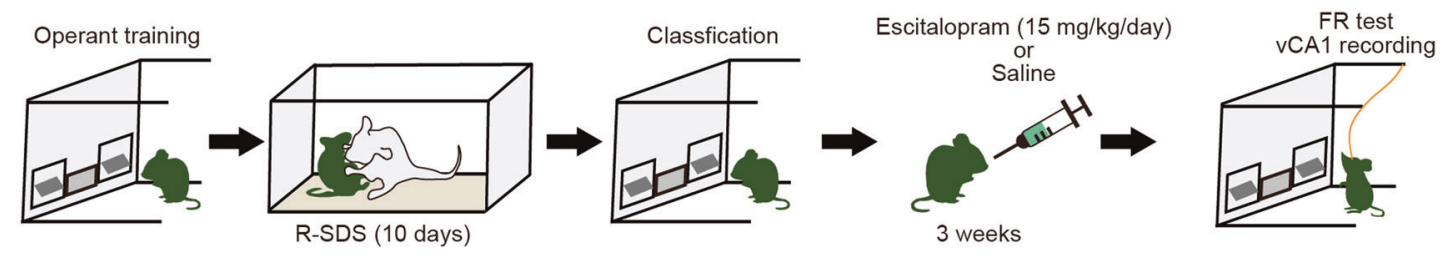

b
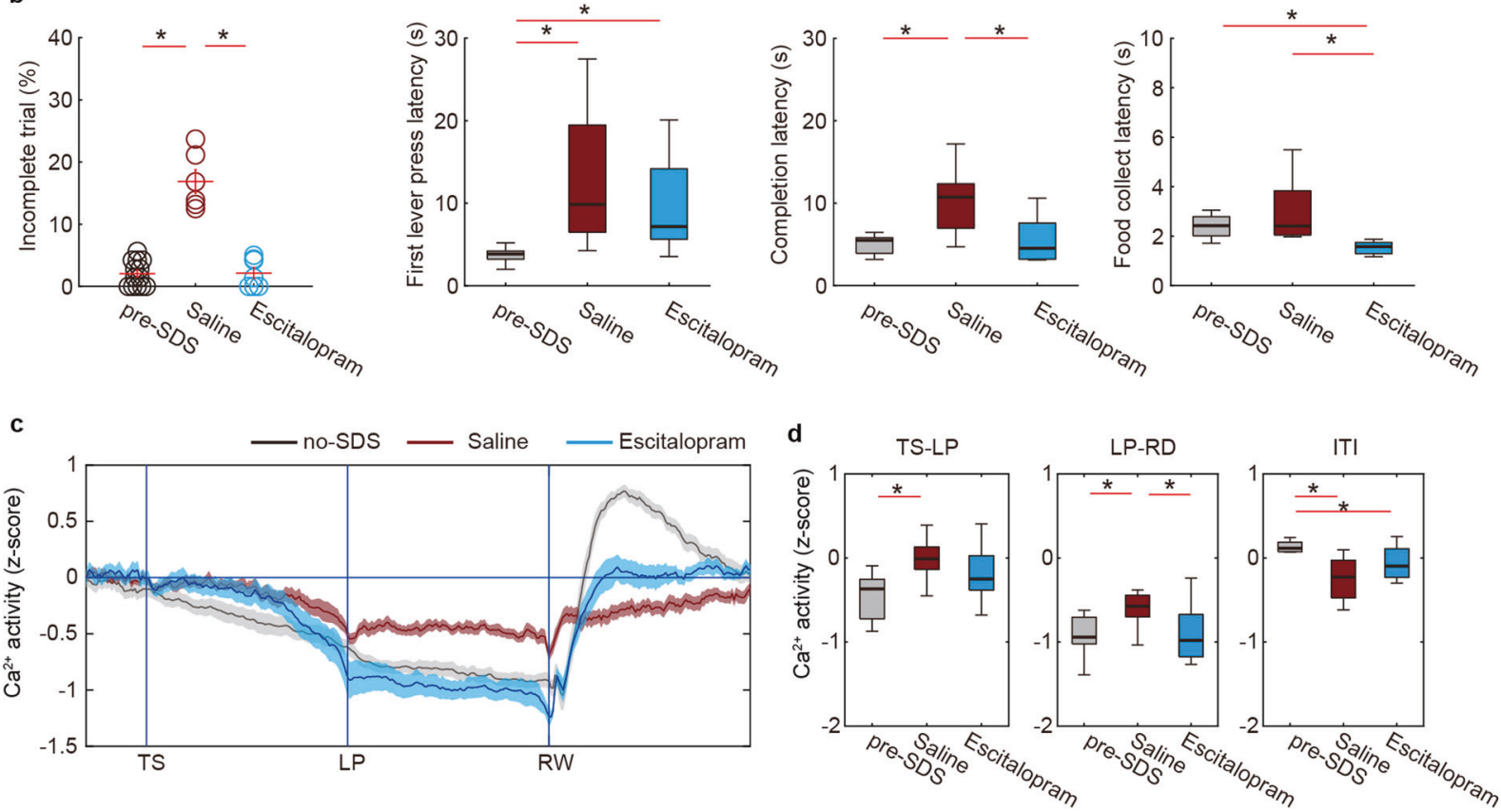

Fig. 3 Chronic SSRI treatment normalized both motivation and ventral hippocampal activity after stress. a Design of the experiment. b The effects of chronic escitalopram treatment on behavior (pre-SDS, $n=13$ mice; saline, $n=6$ mice, escitalopram, $n=7$ mice) in the FR- 5 task. ${ }^{*} p<0.05$ (Mann-Whitney U-test with FDR correction). c, d The effect of chronic escitalopram treatment on Ca ${ }^{2+}$ activity in vCA1 during the FR-5 task (pre-SDS, $n=13$ mice; saline, $n=6$ mice, escitalopram, $n=7$ mice). Trace of averaged $\mathrm{Ca}^{2+}$ signals in which the duration between trigger points was normalized (c). Boxplot representing the averaged $\mathrm{Ca}^{2+}$ signal during TS-LP, LP-RD, and ITI periods of (c), respectively (d). ${ }^{*} p<0.05$ (Mann-Whitney $U$-test with FDR correction). Bars represent the mean and lines represent the s.e.m. The shaded areas represent s.e.m. In box plots, the central mark indicates the median and the bottom and top edges of the box indicate the 25 th and 75 th percentiles, respectively. Whiskers denote the range.

of vCA1 activity only in mice classified as stress-susceptible based on incomplete trial percentage (Fig. 2e, f, brown trace). Among these mice, vCA1 compound $\mathrm{Ca}^{2+}$ levels did not decline during the TS-LP period, the reduction of $\mathrm{Ca}^{2+}$ levels during the LP-RD period was less pronounced than in no-SDS controls, and the rise of $\mathrm{Ca}^{2+}$ levels after RD was attenuated (Fig. 2e, f). Of note, an insufficient reduction of vCA1 activity during lever pressing was previously associated with prolonged latency to task completion [20]. The vCA1 activity of resilient mice was comparable to that of no-SDS controls. These results indicate that task-related vCA1 hyperactivity coincides with stress-induced impairment of successful goal-directed behavior.

Next, we asked whether stress-induced social withdrawal is related to the motivational impairments we observed. We classified the same R-SDS-exposed mice as resilient or susceptible based on $\mathrm{SI}$ ratio and then compared motivated behavior and vCA1 compound $\mathrm{Ca}^{2+}$ levels between these two groups. Surprisingly, goal-directed behavior did not differ between mice that were resilient to social withdrawal and those that were susceptible to social withdrawal (Supplementary Fig. S6). In addition, there were no differences between these two groups in VCA1 activity during the LP-RD period of the operant task (Supplementary Fig. S6). These data suggest that stress-induced motivational impairments are mechanistically distinct from susceptibility to social withdrawal.
Antidepressants restore both vCA1 activity dynamics and motivation after stress

We next addressed whether R-SDS-induced motivation deficit could be treated using antidepressant drugs. After chronic stress exposure and classification of mice based on the percentage of incomplete trials, we administered escitalopram (selective serotonin reuptake inhibitor [SSRI]) to susceptible mice for 3 weeks (Fig. 3a). During SSRI treatment, we monitored behavioral performance on the FR-5 task. We found that 3 weeks of SSRI treatment restored both the trial completion percentage and the completion latency but not the latency to the first LP in the FR-5 task (Fig. 3b), suggesting that SSRI targeted an action sustainment component of the behavior. We therefore monitored the corresponding vCA1 patterns during the behavioral task and found that chronic SSRI treatment restored the suppression of vCA1 activity during lever pressing (LP-RD period) (Fig. 3c, d), suggesting that the therapeutic effect of SSRI was mediated by the normalization of vCA1 activity during sustained goal-directed action.

Recent clinical studies confirm that ketamine is effective as a rapidly acting antidepressant [37-39]. In rodents, ketamine is effective against R-SDS-induced depression-like behavior [40]. We sought to address whether ketamine could ameliorate stressinduced motivation impairments and normalize vCA1 activity, similar to chronic SSRI treatment. We administered a single ketamine injection with a subanesthetic dose to susceptible mice 


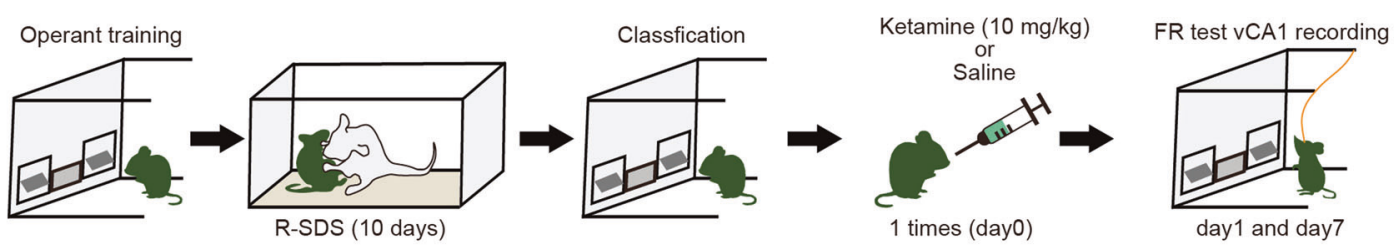

b
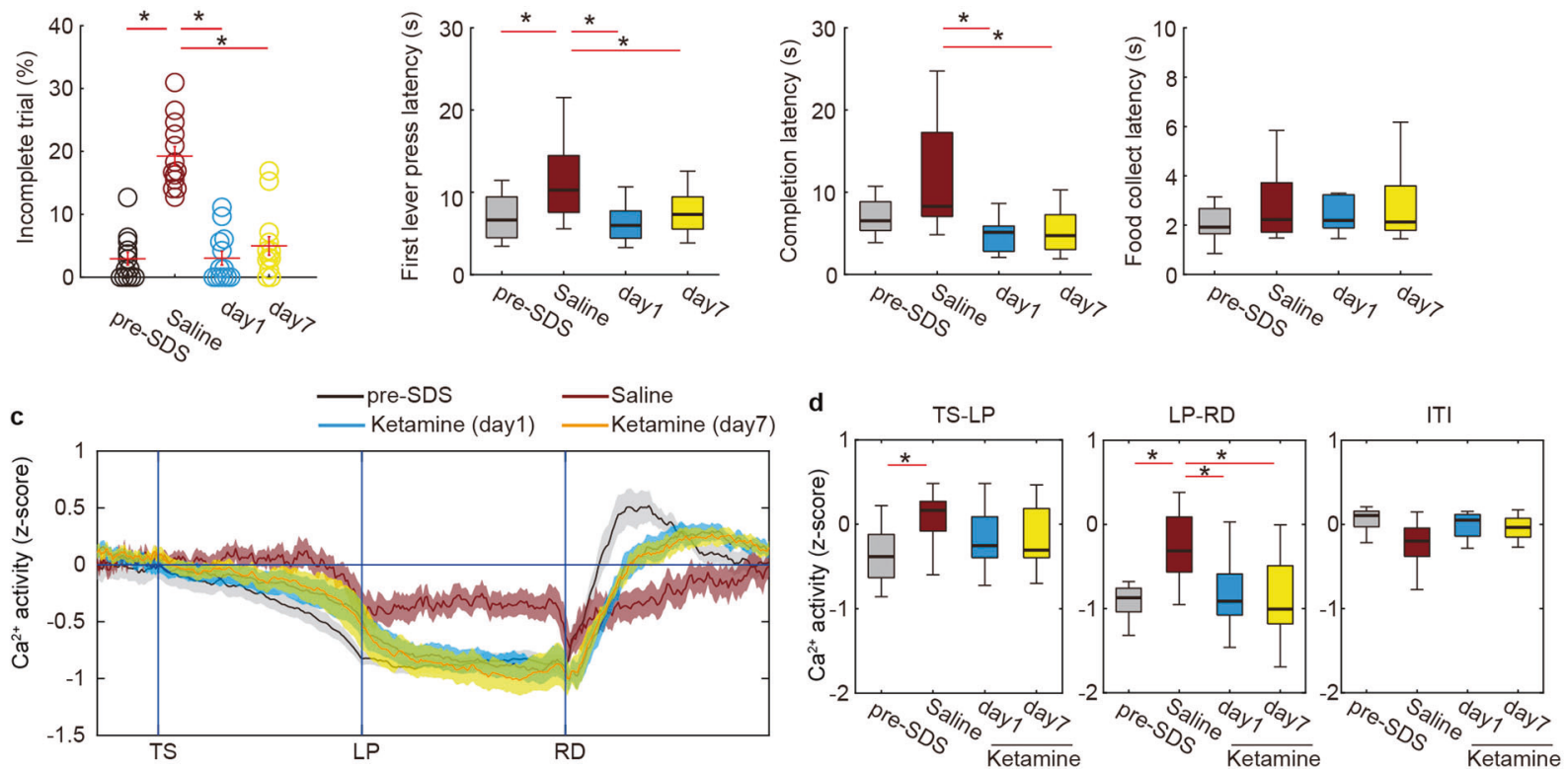

Fig. 4 Rapid and long-term effects of a single ketamine injection on motivation and ventral hippocampal activity after stress. a Schematic illustration of ketamine treatment. $\mathbf{b}$ The effects of ketamine treatment on behavior (base, $n=13$ mice; saline, $n=13$ mice; ketamine (day 1), $n=13$ mice; ketamine (day 7), $n=13$ mice) in the FR-5 task. ${ }^{*} p<0.05$ (Mann-Whitney U-test with FDR correction). c, d The effect of ketamine treatment on $\mathrm{Ca}^{2+}$ activity in vCA1 during the FR-5 task (base, $n=13$ mice; saline, $n=13$ mice; ketamine (day 1 ), $n=13$ mice; ketamine (day 7), $n=13$ mice). Trace of averaged $\mathrm{Ca}^{2+}$ signals in which the duration between trigger points was normalized (g). Boxplot representing the averaged $\mathrm{Ca}^{2+}$ signal during TS-LP, LP-RD, and ITI periods of (c), respectively (h). ${ }^{*} p<0.05$ (Mann-Whitney U-test with FDR correction). Bars represent the mean and lines represent the s.e.m. The shaded areas represent s.e.m. In box plots, the central mark indicates the median and the bottom and top edges of the box indicate the 25th and 75th percentiles, respectively. Whiskers denote the range.

and monitored behavioral performance and vCA1 activity during the FR-5 task 1 and 7 days after the treatment (Fig. 4a). At both time points, a single ketamine treatment restored all behavioral parameters that had been altered after R-SDS (Fig. 4b), demonstrating rapid and long-lasting effects of ketamine in our mouse model. Notably, ketamine rescued deficits in both action initiation and sustainment. During action sustainment (LP-RD period), ketamine reversed the stress-induced increase in VCA1 activity at both 1 and 7 days after treatment (Fig. 4c, d), suggesting that the behavioral effect of ketamine was mediated by modulation vCA1 activity dynamics.

To address whether ketamine modulates motivated behavior under unstressed conditions, we trained mice to press lever to earn food on a FR-10 schedule, in which more effort was required and, as a consequence, the incomplete trial percentage was higher than in the FR-5. We administered a single ketamine injection to unstressed mice and monitored behavior and vCA1 activity. There were no significant effects of ketamine on behavior or VCA1 activity under either the FR-10 or FR-5 schedules (Supplementary Fig. S7). These data indicate that the effects of ketamine are unique to stressed animals.

The SSRI and ketamine experiments suggest normalization of vCA1 activity during lever pressing may be a shared therapeutic mechanism for stress-induced motivation impairments. To test this hypothesis, we investigated whether temporally specific augmentation of vCA1 suppression is sufficient to rescue impairments in goal-directed behavior caused by R-SDS. For vCA1 inhibition, we used transgenic mice (Htr5B-ArchT mice), in which only the CA1 pyramidal neurons expressed an inhibitory opsin (ArChT) (Fig. 5a, supplementary Fig. S8). After stress exposure, susceptible mice were identified as above based on the percentage of incomplete trials. We then evaluated the effects of bilateral optogenetic inhibition of vCA1 activity on FR-5 task performance (Fig. 5b, c). Inhibition of VCA1 during the LP-RD period reduced the percentage of incomplete trials and the completion latency in R-SDS-exposed mice (Fig. 5d, e). This manipulation did not affect latency to the first LP or latency to food collection (Fig. 5e), confirming prior evidence for a specific role of vCA1 in action sustainment [20]. Inhibition of VCA1 during the TS-LP period did not affect behavioral parameters (Fig. 5f, g), emphasizing the temporal specificity of vCA1 suppression. vCA1 inhibition did not increase locomotor activity (Supplementary Fig. S9), suggesting that the increase in motivated behavior was not due to hyperlocomotion. Taken together, our results suggest that R-SDS prevents vCA1 suppression during sustained goal-directed behavior, thereby impairing of action sustainment and reducing success of goal-directed behavior. After stress, chronic SSRI treatment or a single ketamine injection restores motivated behavior via normalization of vCA1 activity during action sustainment.

\section{DISCUSSION}

Our results demonstrate that chronic stress exposure impairs goaldirected motivated behavior in mice. During 10 days of R-SDS, 
a

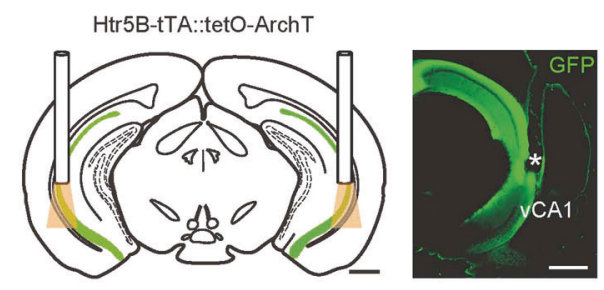

b Experimental schedule

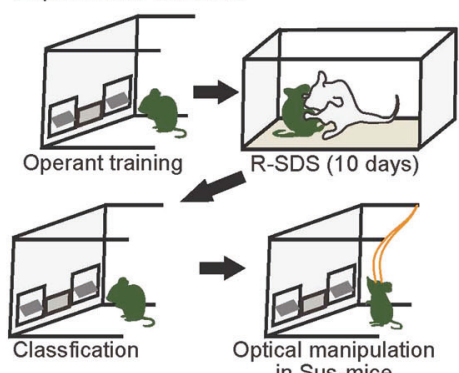

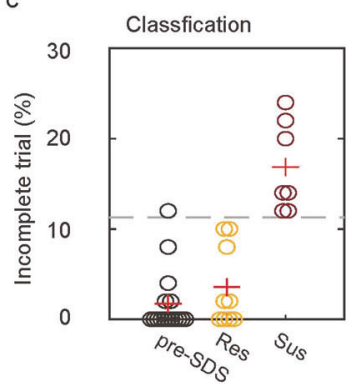

d

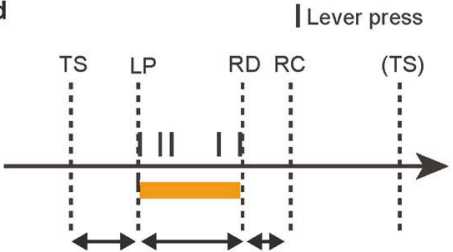

(i)

(ii) (iii)

f

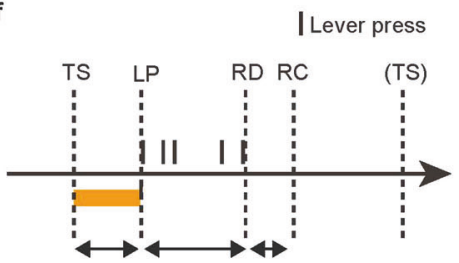

(i) (ii) (iii)
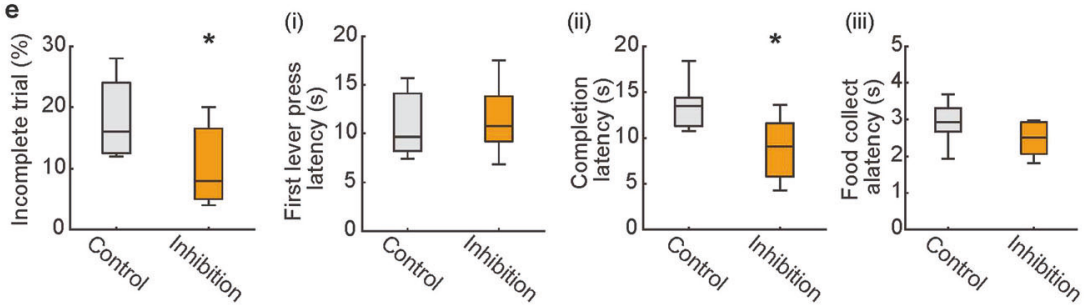

Fig. 5 Artificial suppression of vCA1 activity rescues stress-induced motivational impairments. a Bilateral optogenetic inhibition of vCA1 was performed in Htr5B-ArchT mice (left). Expression of ArchT-EGFP in vCA1 of Htr5B-ArchT mouse. The white asterisk denotes optical fiber placement. Scale bar, $1 \mathrm{~mm}$. b Design of optogenetic experiment. c Defeated mice $(n=16$ mice) were categorized into resilient ( $n=9)$ or susceptible $(n=7)$ based on incomplete trials (Threshold is $11.3 \%$, gray dashed line). d, f Timing of illumination. Optogenetic inhibition was conducted during every LP-RD (d) or TS-LP (f) period. e The effects of VCA1 inhibition in susceptible mice during the LP-RD period of the FR-5 task $\left(n=7\right.$ mice). ${ }^{*} p<0.05$, two-sided Wilcoxon signed-rank test. $\mathbf{g}$ The effects of vCA1 inhibition in susceptible mice during the TS-LP period

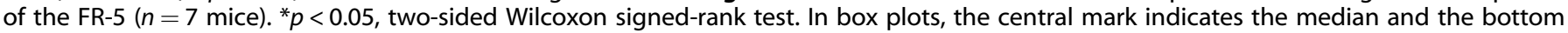
and top edges of the box indicate the 25 th and 75 th percentiles, respectively. Whiskers denote the range.

mice lever pressing for food RW displayed increases in the number of incomplete trials, the latency to start lever pressing, and the latency to complete a trial. In contrast, the latency to collect the food RWs, once earned, was unaffected by stress, suggesting that motivational deficits do not reflect blunted hedonic RW. Stressinduced motivational impairments were associated with hyperactivity of the vHP during periods when effortful responding was required. The behavioral deficits and hippocampal hyperactivity were corrected by each of two antidepressant medications, the SSRI escitalopram and ketamine.

The hippocampus is believed to play important roles in the stress response and the pathology of depression [18, 41]. Hyperactivity of vHP is associated with negative-valence states such as fear and anxiety, and hyperactivity of vHP impairs goaldirected behavior [20]. Stress-exposed rodents show increased glutamate release [42], increased glutamate receptor expression [43], and facilitated induction of c-fos and Arc mRNA expression in the hippocampus [44]. Artificial enhancement of glutamatergic transmission from vHP to nucleus accumbens induced susceptible phenotype after chronic defeat stress [45]. The ventral extent of the hippocampus is particularly sensitive to the effects of stress [15-17]. These observations may account for the hyperactivity of vHP after stress exposure and the insufficient decline of vHP activity during goal-directed behaviors.

Our data demonstrate that vHP dysregulation plays a direct causal role in stress-induced motivational deficits and that the vHP activity is a shared target of antidepressants of different classes. The suppressive effects of these drugs on VHP activity may be mediated by direct or indirect serotoninmediated inhibition [20,46], ketamine-mediated NMDA receptor blockade $[37,40]$, or ketamine-mediated serotonin uptake inhibition [47]; however, more detailed investigations will be necessary to explain the temporal specificity of the suppressive effects.

A recent study demonstrated that RW behavior is modulated by the strength of vHP to nucleus accumbens synapses [48]. In that study, chronic stress reduced the strength of vHP-accumbal dopamine receptor type1-expressing medium spiny neuron (D1MSN) synapses but not D2-MSN synapses, and SSRI treatment rescued it [48]. Our data demonstrate vHP hyperactivity under chronic stress exposure, but why bulk vHP hyperactivity would selectively weaken vHP-accumbal D1-MSN synaptic strength is unclear. One possibility is that the bulk $\mathrm{Ca}^{2+}$ activity that we monitored is insensitive to differences in activity among specific projecting populations (e.g., D1-targeting versus D2-targeting vCA1 cells). It will be valuable to monitor projection-specific neural activity during motivated behavior to determine whether changes in activity dynamics are confined to particular projection pathways. Although the two studies highlight different circuit mechanisms, the LeGate et al. [48] study and our own converge on VCA 1 as an important target for understanding stress and antidepressant effects on motivated behavior. 
Our results suggest that acute and chronic stress have distinct motivational effects. On day 1 after stress exposure, mice exhibited prolonged latency to the first LP, while measures of action sustainment remained were unaffected. The latency to the first LP reflects the action initiation component in effortful goaldirected behaviors [31] and is modulated by an insular cortexventral striatum pathway [35]. Interestingly, stress-induced increases in first LP latency were responsive to ketamine treatment (Fig. 5b) but not SSRI treatment (Fig. 4b). These pharmacological responses suggest that an acute-onset and sustained action initiation impairment is not mediated by the dysregulation of the ventral hippocampal activity. Insular cortex is known to mediate the retrieval of the outcome value [49] and to guide behaviors on the basis of anticipation of food [50]. These processes may preferentially be affected by acute stress and impair initiation of goal-directed behaviors. Further studies are needed to dissociate circuitries targeted by acute and chronic stress.

Because multiple systems-endocrine, immune, autonomic, emotional, and cognitive-are involved in the stress response, it is plausible that stress resilience and susceptibility are multidimensional processes with the potential for orthogonal outcomes at each of several potentially stress-responsive loci. Social withdrawal is one well-known sequela of R-SDS, and numerous studies have validated the susceptible vs resilient dichotomy with respect to this outcome in the R-SDS model [51-56]. Our data suggest that impaired goal-directed motivation constitutes an independent dimension of the stress response, and susceptibility and resilience at the level of motivation is orthogonal to an individual's classification with respect to social behavior. We hypothesize that stress-induced social and motivational impairments have distinct circuit mechanisms, with a vHP-mediated emotional and cognitive system shaping responsiveness in the motivational dimension (our study) and a PFC/amygdala-mediated system controlling that in the social dimension $[32,57]$ in the R-SDS model. Our findings favor the view that effective treatments for stress-related psychopathology will require treatment approaches tailored to an individual's particular pattern of susceptible and resilient behavioral domains and their associated neural circuits.

In addition to establishing a link between stress and goaldirected behavior, the paradigm established here may have general utility as a model for investigating treatments and mechanisms of motivational disorders. According to the framework put forth by Nestler and Hyman [58], animal models of neuropsychiatric conditions should be evaluated by reference to three forms of validity. Face validity of the current model derives from the fact that the operant lever pressing-deficits task is readily translatable to humans, and, in fact, similar lever pressing impairments have been observed in patients with major depression [8]. Pharmacological validity refers to a model's ability to accurately discriminate effective versus ineffective therapeutics. Although we have not established that the current model can identify novel antidepressant therapeutics, which is the strongest test of pharmacological validity, we are encouraged by the fact that the current model is sensitive to two antidepressants of different classes. Construct validity refers to homology between the environmental or biological conditions that produce the disease. In humans, stressful life events have a well-established causal effect on depression onset [23]. As for biological constructs, it is unknown whether task-related vHP hyperactivity of the kind reported here is present in human patients, but this phenotype is amenable to analysis in humans, provided that imaging modalities such as fMRI or PET can provide adequate temporal precision.

One caveat of this experiment is that calorie restriction by itself can produce anti-depressive effects [59]. Since food restriction is required for good performance in our operant task, it would be difficult to investigate whether food restriction moderated the effects of stress in this task. However, we can conclude that food restriction was not sufficient to prevent effects of R-SDS on motivation, nor was it sufficient to prevent the effects of our antidepressant manipulations. A related possibility is that changes in operant performance in our task reflect changes in hunger/ satiety. This is unlikely, however, because food collection latency was not affected by our manipulations, indicating that mice still desired the food when it was available.

In conclusion, our data identify vCA1 activity dynamics as a putative biomarker for stress-induced motivational deficits. vCA1 activity differentiates susceptible versus resilient individuals and is responsive to antidepressant treatment. Furthermore, optogenetic stimulation demonstrates a causal effect of vHP activity on goaldirected behavior. The fact that $\mathrm{VCA} 1$ activity responded to antidepressant drugs with distinct primary pharmacological targets suggests that vCA1 is a higher-order therapeutic target that might be modulated via various signaling pathways. The results encourage the use of vCA1 dynamics as a circuit-level biomarker that might be used to accelerate discovery of drugs targeting motivational impairments.

\section{FUNDING AND DISCLOSURE}

This work was supported by Grant for Research Fellow of the Japan Society for the Promotion of Science (20J00643) to KY, Grant-in-Aid for Scientific Research on Innovative Area "Willdynamics" (19H05027) from the MEXT to KFT, Grant-in-Aid for Brain Mapping by Integrated Neurotechnologies for Disease Studies (Brain/MINDS) (JP20dm0207069) from AMED to KFT, and Grant-in-Aid for Program for the Advancement of Next Generation Research Projects from Keio University to KFT. The authors declare no competing interests. We dedicate this paper to the memory of Dr. Keitaro Yoshida, an immensely talented graduate student who passed away while he was spearheading this project.

\section{AUTHOR CONTRIBUTIONS}

$\mathrm{KY}$ conducted all experiments and analyses, AK conducted control behavioral experiments. NT analyzed data. MM and KFT designed the experiments, KY, MRD, KFT wrote the paper.

\section{ADDITIONAL INFORMATION}

Supplementary information The online version contains supplementary material available at https://doi.org/10.1038/s41386-021-00990-y.

Publisher's note Springer Nature remains neutral with regard to jurisdictional claims in published maps and institutional affiliations.

\section{REFERENCES}

1. Calabrese JR, Fava M, Garibaldi G, Grunze $H$, Krystal AD, Laughren $T$, et al. Methodological approaches and magnitude of the clinical unmet need associated with amotivation in mood disorders. J Affect Disord. 2014;168:439-51.

2. Fervaha G, Foussias G, Takeuchi H, Agid O, Remington G. Motivational deficits in major depressive disorder: cross-sectional and longitudinal relationships with functional impairment and subjective well-being. Compr Psychiatry. 2016;66: 31-8.

3. McGlinchey JB, Zimmerman M, Young D, Chelminski I. Diagnosing major depressive disorder VIII: are some symptoms better than others? J Nerv Ment Dis. 2006;194:785-90.

4. Cooper JA, Arulpragasam AR, Treadway MT. Anhedonia in depression: biological mechanisms and computational models. Curr Opin Behav Sci. 2018;22:128-35.

5. Filla I, Bailey MR, Schipani E, Winiger V, Mezias C, Balsam PD, et al. Striatal dopamine D2 receptors regulate effort but not value-based decision making and alter the dopaminergic encoding of cost. Neuropsychopharmacology. 2018;43: 2180-9.

6. Treadway MT, Zald DH. Parsing anhedonia: translational models of rewardprocessing deficits in psychopathology. Curr Dir Psychol Sci. 2013;22:244-9.

7. Thomsen KR. Measuring anhedonia: impaired ability to pursue, experience, and learn about reward. Front Psychol. 2015;6:1409. 
8. Hershenberg R, Satterthwaite TD, Daldal A, Katchmar N, Moore TM, Kable JW, et al. Diminished effort on a progressive ratio task in both unipolar and bipolar depression. J Affect Disord. 2016;196:97-100.

9. Treadway MT, Bossaller NA, Shelton RC, Zald DH. Effort-based decision-making in major depressive disorder: a translational model of motivational anhedonia. J Abnorm Psychol. 2012;121:553-8.

10. Krishnan V, Han MH, Graham DL, Berton O, Renthal W, Russo SJ, et al. Molecular adaptations underlying susceptibility and resistance to social defeat in brain reward regions. Cell. 2007;131:391-404.

11. Willner P, Towell A, Sampson D, Sophokleous S, Muscat R. Reduction of sucrose preference by chronic unpredictable mild stress, and its restoration by a tricyclic antidepressant. Psychopharmacology. 1987;93:358-64.

12. Ironside $M$, Kumar $P$, Kang MS, Pizzagalli DA. Brain mechanisms mediating effects of stress on reward sensitivity. Curr Opin Behav Sci. 2018;22:106-13.

13. Kukel'ova D, Bergamini G, Sigrist H, Seifritz E, Hengerer B, Pryce CR. Chronic socia stress leads to reduced gustatory reward salience and effort valuation in mice. Front Behav Neurosci. 2018;12:134.

14. Bergamini G, Cathomas F, Auer S, Sigrist H, Seifritz E, Patterson M, et al. Mouse psychosocial stress reduces motivation and cognitive function in operant reward tests: A model for reward pathology with effects of agomelatine. Eur Neuropsychopharmacol. 2016;26:1448-64.

15. Maggio N, Segal M. Striking variations in corticosteroid modulation of long-term potentiation along the septotemporal axis of the hippocampus. J Neurosci. 2007;27:5757-65.

16. Pinto V, Costa JC, Morgado P, Mota C, Miranda A, Bravo FV, et al. Differential impact of chronic stress along the hippocampal dorsal-ventral axis. Brain Struct Funct. 2015;220:1205-12.

17. Floriou-Servou A, von Ziegler L, Stalder L, Sturman O, Privitera M, Rassi A, et al Distinct proteomic, transcriptomic, and epigenetic stress responses in dorsal and ventral hippocampus. Biol Psychiatry. 2018;84:531-41.

18. Anacker C, Luna VM, Stevens GS, Millette A, Shores R, Jimenez JC, et al. Hippocampal neurogenesis confers stress resilience by inhibiting the ventral dentate gyrus. Nature. 2018;559:98-102.

19. O'Leary OF, Cryan JF. A ventral view on antidepressant action: roles for adult hippocampal neurogenesis along the dorsoventral axis. Trends Pharmacol Sci 2014;35:675-87.

20. Yoshida K, Drew MR, Mimura M, Tanaka KF. Serotonin-mediated inhibition of ventral hippocampus is required for sustained goal-directed behavior. Nat Neurosci. 2019;22:770-7.

21. Fee $C$, Prevot $T$, Misquitta $K$, Banasr M, Sibille E. Chronic stress-induced behaviors correlate with exacerbated acute stress-induced cingulate cortex and ventral hippocampus activation. Neuroscience. 2020;440:113-29.

22. Matsuda S, Peng H, Yoshimura H, Wen TC, Fukuda T, Sakanaka M. Persistent c-fos expression in the brains of mice with chronic social stress. Neurosci Res. 1996;26:157-70.

23. Kendler KS, Karkowski LM, Prescott CA. Causal relationship between stressful life events and the onset of major depression. Am J Psychiatry. 1999;156: 837-41.

24. Feder A, Fred-Torres S, Southwick SM, Charney DS. The biology of human resilience: opportunities for enhancing resilience across the life span. Biol Psychiatry. 2019:86:443-53.

25. Hodes GE, Pfau ML, Leboeuf M, Golden SA, Christoffel DJ, Bregman D, et al. Individual differences in the peripheral immune system promote resilience versus susceptibility to social stress. Proc Natl Acad Sci USA 2014;111: 16136-41.

26. Nasca C, Menard C, Hodes G, Bigio B, Pena C, Lorsch Z, et al. Multidimensional predictors of susceptibility and resilience to social defeat stress. Biol Psychiatry. 2019;86:483-91.

27. Tanaka KF, Matsui K, Sasaki T, Sano H, Sugio S, Fan K, et al. Expanding the repertoire of optogenetically targeted cells with an enhanced gene expression system. Cell Rep. 2012;2:397-406.

28. Kanemaru K, Sekiya H, Xu M, Satoh K, Kitajima N, Yoshida K, et al. In vivo visualization of subtle, transient, and local activity of astrocytes using an ultrasensitive $\mathrm{Ca}(2+)$ indicator. Cell Rep. 2014;8:311-8.

29. Tsunematsu T, Tabuchi S, Tanaka KF, Boyden ES, Tominaga M, Yamanaka A. Longlasting silencing of orexin/hypocretin neurons using archaerhodopsin induces slow-wave sleep in mice. Behav Brain Res. 2013;255:64-74.

30. Paxinos G, Franklin KBJ. Paxinos and Franklin's the mouse brain in stereotaxic coordinates, compact. San Diego, Elsevier Academic Press; 2019.

31. Natsubori A, Tsutsui-Kimura I, Nishida $H$, Bouchekioua $Y$, Sekiya $H$, Uchigashima $M$, et al. Ventrolateral striatal medium spiny neurons positively regulate foodincentive, goal-directed behavior independently of D1 and D2 selectivity. J Neurosci. 2017;37:2723-33.
32. Nie X, Kitaoka S, Tanaka K, Segi-Nishida E, Imoto $Y$, Ogawa A, et al. The innate immune receptors TLR2/4 mediate repeated social defeat stress-induced social avoidance through prefrontal microglial activation. Neuron. 2018;99: 464-79 e7.

33. Sanchez C, Bergqvist PB, Brennum LT, Gupta S, Hogg S, Larsen A, et al. Escitalopram, the S-(+)-enantiomer of citalopram, is a selective serotonin reuptake inhibitor with potent effects in animal models predictive of antidepressant and anxiolytic activities. Psychopharmacology 2003;167:353-62.

34. Brachman RA, McGowan JC, Perusini JN, Lim SC, Pham TH, Faye C, et al. Ketamine as a prophylactic against stress-induced depressive-like behavior. Biol Psychiatry. 2016;79:776-86.

35. Yoshida K, Tsutsui-Kimura I, Kono A, Yamanaka A, Kobayashi K, Watanabe M, et al Opposing ventral striatal medium spiny neuron activities shaped by striatal parvalbumin-expressing interneurons during goal-directed behaviors. Cell Rep. 2020;31:107829.

36. Berton O, McClung CA, Dileone RJ, Krishnan V, Renthal W, Russo SJ, et al. Essential role of BDNF in the mesolimbic dopamine pathway in social defeat stress. Science. 2006;311:864-8.

37. Berman RM, Cappiello A, Anand A, Oren DA, Heninger GR, Charney DS, et al. Antidepressant effects of ketamine in depressed patients. Biol Psychiatry. 2000 47:351-4.

38. Kishimoto T, Chawla JM, Hagi K, Zarate CA, Kane JM, Bauer M, et al. Single-dose infusion ketamine and non-ketamine $\mathrm{N}$-methyl-d-aspartate receptor antagonists for unipolar and bipolar depression: a meta-analysis of efficacy, safety and time trajectories. Psychol Med. 2016;46:1459-72.

39. Newport DJ, Carpenter LL, McDonald WM, Potash JB, Tohen M, Nemeroff CB, et al. Ketamine and other NMDA antagonists: early clinical trials and possible mechanisms in depression. Am J Psychtry. 2015;172:950-66.

40. Zanos P, Moaddel R, Morris PJ, Georgiou P, Fischell J, Elmer Gl, et al. NMDAR inhibition-independent antidepressant actions of ketamine metabolites. Nature. 2016:533:481-6.

41. Joels M, Baram TZ. The neuro-symphony of stress. Nat Rev Neurosci. 2009;10: 459-66.

42. Moghaddam B, Bolinao ML, Stein-Behrens B, Sapolsky R. Glucocorticoids mediate the stress-induced extracellular accumulation of glutamate. Brain Res. 1994;655: 251-4.

43. Chang $\mathrm{CH}$, Hsiao YH, Chen YW, Yu YJ, Gean PW. Social isolation-induced increase in NMDA receptors in the hippocampus exacerbates emotional dysregulation in mice. Hippocampus. 2015;25:474-85.

44. Pacheco A, Aguayo Fl, Aliaga E, Munoz M, Garcia-Rojo G, Olave FA, et al. Chronic stress triggers expression of immediate early genes and differentially affects the expression of AMPA and NMDA subunits in dorsal and ventral hippocampus of rats. Front Mol Neurosci. 2017;10:244.

45. Bagot RC, Parise EM, Pena CJ, Zhang HX, Maze I, Chaudhury D, et al. Ventral hippocampal afferents to the nucleus accumbens regulate susceptibility to depression. Nat Commun. 2015;6:7062.

46. Wang DV, Yau HJ, Broker CJ, Tsou JH, Bonci A, Ikemoto S. Mesopontine median raphe regulates hippocampal ripple oscillation and memory consolidation. Nat Neurosci. 2015;18:728-35.

47. Bowman MA, Vitela M, Clarke KM, Koek W, Daws LC. Serotonin transporter and plasma membrane monoamine transporter are necessary for the antidepressantlike effects of ketamine in mice. Int J Mol Sci. 2020;21:7581-7602.

48. LeGates TA, Kvarta MD, Tooley JR, Francis TC, Lobo MK, Creed MC, et al. Reward behaviour is regulated by the strength of hippocampus-nucleus accumbens synapses. Nature. 2018;564:258-62.

49. Parkes SL, Bradfield LA, Balleine BW. Interaction of insular cortex and ventral striatum mediates the effect of incentive memory on choice between goaldirected actions. J Neurosci. 2015;35:6464-71.

50. Kusumoto-Yoshida I, Liu H, Chen BT, Fontanini A, Bonci A. Central role for the insular cortex in mediating conditioned responses to anticipatory cues. Proc Nat Acad Sci USA. 2015;112:1190-5.

51. Chaudhury D, Walsh JJ, Friedman AK, Juarez B, Ku SM, Koo JW, et al. Rapid regulation of depression-related behaviours by control of midbrain dopamine neurons. Nature. 2013;493:532-6.

52. Hultman R, Ulrich K, Sachs BD, Blount C, Carlson DE, Ndubuizu N, et al. Brain-wide electrical spatiotemporal dynamics encode depression vulnerability. Cell. 2018;173:166-80 e14.

53. Tye KM, Mirzabekov JJ, Warden MR, Ferenczi EA, Tsai HC, Finkelstein J, et al Dopamine neurons modulate neural encoding and expression of depressionrelated behaviour. Nature. 2013;493:537-41.

54. Knowland D, Lilascharoen V, Pacia CP, Shin S, Wang EH, Lim BK. Distinct ventral pallidal neural populations mediate separate symptoms of depression. Cell. 2017;170:284-97 e18. 
Chronic social defeat stress impairs goal-directed behavior through...

$\mathrm{K}$ Yoshida et al.

1616

55. Isingrini E, Perret L, Rainer Q, Amilhon B, Guma E, Tanti A, et al. Resilience to chronic stress is mediated by noradrenergic regulation of dopamine neurons. Nat Neurosci. 2016;19:560-3.

56. Christoffel DJ, Golden SA, Walsh JJ, Guise KG, Heshmati M, Friedman AK, et al. Excitatory transmission at thalamo-striatal synapses mediates susceptibility to social stress. Nat Neurosci. 2015;18:962-4.

57. Kumar S, Hultman R, Hughes D, Michel N, Katz BM, Dzirasa K. Prefrontal cortex reactivity underlies trait vulnerability to chronic social defeat stress. Nat Commun. 2014;5:4537.

58. Nestler EJ, Hyman SE. Animal models of neuropsychiatric disorders. Nat Neurosci. 2010;13:1161-9.

59. Lutter M, Nestler EJ. Homeostatic and hedonic signals interact in the regulation of food intake. J Nutr. 2009;139:629-32.
Open Access This article is licensed under a Creative Commons Attribution 4.0 International License, which permits use, sharing, adaptation, distribution and reproduction in any medium or format, as long as you give appropriate credit to the original author(s) and the source, provide a link to the Creative Commons license, and indicate if changes were made. The images or other third party material in this article are included in the article's Creative Commons license, unless indicated otherwise in a credit line to the material. If material is not included in the article's Creative Commons license and your intended use is not permitted by statutory regulation or exceeds the permitted use, you will need to obtain permission directly from the copyright holder. To view a copy of this license, visit http://creativecommons. org/licenses/by/4.0/.

(c) The Author(s) 2021 